\title{
On meromorphic solutions of some linear differential equations with entire coefficients being Fabry gap series
}

Shun-Zhou Wu and Xiu-Min Zheng*

"Correspondence:

zhengxiumin2008@sina.com Institute of Mathematics and Information Science, Jiangxi Normal University, Nanchang, Jiangxi 330022, P.R. China

\section{Abstract}

In this paper, we investigate the growth and the exponent of convergence of the sequence of $\varphi$-points of meromorphic solutions of the linear differential equations

$$
A_{k}(z) f^{(k)}+A_{k-1}(z) f^{(k-1)}+\cdots+A_{1}(z) f^{\prime}+A_{0}(z) f=0
$$

and

$$
A_{k}(z) f^{(k)}+A_{k-1}(z) f^{(k-1)}+\cdots+A_{1}(z) f^{\prime}+A_{0}(z) f=F(z),
$$

with entire coefficients $A_{j}(z), j=0,1, \ldots, k$ and $F(z)$, where $k \geq 2, A_{0}(z) A_{k}(z) \not \equiv 0, \varphi(z)$ is a meromorphic function of finite order, and there is only one dominant coefficient $A_{k}(z)$ of the maximal order, which is also a Fabry gap series.

MSC: 30D35; 34M10

Keywords: linear differential equation; meromorphic solution; growth; exponent of convergence; Fabry gap series

\section{Introduction and main results}

We make use of the standard notations of Nevanlinna's value distribution theory (see, e.g., [1-3]). In the whole paper, let $f(z)$ be a meromorphic function in the whole complex plane.

Firstly, let us recall the following definitions (see, e.g., [4-6]).

Definition 1.1 The iterated $p$-order $\sigma_{p}(f)$ and the iterated $p$-lower order $\mu_{p}(f)$ of a meromorphic function $f(z)$ are defined respectively by

$$
\sigma_{p}(f)=\varlimsup_{r \rightarrow \infty} \frac{\log _{p} T(r, f)}{\log r} \quad \text { and } \quad \mu_{p}(f)=\varliminf_{r \rightarrow \infty} \frac{\log _{p} T(r, f)}{\log r}, \quad p \in \mathbb{N}
$$

Especially, $\sigma(f)=\sigma_{1}(f), \mu(f)=\mu_{1}(f)$.

\section{Springer}

( 2015 Wu and Zheng; licensee Springer. This is an Open Access article distributed under the terms of the Creative Commons Attribution License (http://creativecommons.org/licenses/by/4.0), which permits unrestricted use, distribution, and reproduction in any medium, provided the original work is properly credited. 
Definition 1.2 The finiteness degree of the order of a meromorphic function $f(z)$ is defined by

$$
i(f)= \begin{cases}0 & \text { if } f(z) \text { is rational; } \\ \min \left\{p \in \mathbb{N}: \sigma_{p}(f)<\infty\right\} & \text { if } f(z) \text { is transcendental with } \\ & \sigma_{p}(f)<\infty \text { for some } p \in \mathbb{N} \\ \infty & \text { if } \sigma_{p}(f)=\infty \text { for all } p \in \mathbb{N} .\end{cases}
$$

Definition 1.3 The iterated exponents of convergence of the sequence of $a$-points and the sequence of distinct $a$-points of a meromorphic function $f(z)$ are defined respectively by

$$
\lambda_{p}(f-a)=\varlimsup_{r \rightarrow \infty} \frac{\log _{p} N\left(r, \frac{1}{f-a}\right)}{\log r} \quad \text { and } \quad \bar{\lambda}_{p}(f-a)=\varlimsup_{r \rightarrow \infty} \frac{\log _{p} \bar{N}\left(r, \frac{1}{f-a}\right)}{\log r}, \quad p \in \mathbb{N},
$$

where $a \in \mathbb{C} \cup\{\infty\}$.

Further, we can get the definitions $\lambda_{p}(f-\varphi)$ and $\bar{\lambda}_{p}(f-\varphi)$, when $a$ is replaced by a meromorphic function $\varphi(z)$.

Secondly, let us recall some results on the growth of solutions of the homogeneous linear differential equation

$$
f^{(k)}+A_{k-1}(z) f^{(k-1)}+\cdots+A_{1}(z) f^{\prime}+A_{0}(z) f=0,
$$

where $k \geq 2, A_{j}(z), j=0,1, \ldots, k-1$, are entire functions (see, e.g., [4, 6-13]). It is well known that all solutions of (1.1) are entire functions.

In 1993, Chen and Gao considered the growth of solutions of (1.1) and obtained the following theorem in [9].

Theorem A (see [9]) Suppose that $k \geq 2, A_{j}(z), j=0,1, \ldots, k-1$, are entire functions and satisfy

(i) $\sigma\left(A_{j}\right)<\sigma\left(A_{0}\right)<\infty, j=1, \ldots, k-1$

or

(ii) $A_{0}(z)$ is a transcendental entire function with $\sigma\left(A_{0}\right)<\infty$, and $A_{j}(z), j=1, \ldots, k-1$, are polynomials.

Then every solution $f(z)(\not \equiv 0)$ of $(1.1)$ satisfies $\sigma(f)=\infty$.

Generally, when $A_{d}(z)(0 \leq d \leq k-1)$ is dominant, Chen and Gao obtained the following theorem in [10] in 1997.

Theorem B (see [10]) Suppose that $k \geq 2, A_{j}(z), j=0,1, \ldots, k-1$, are entire functions, and there exists $A_{d}(z)(0 \leq d \leq k-1)$ such that

(i) $\sigma\left(A_{j}\right)<\sigma\left(A_{d}\right)<\frac{1}{2}(j=0, \ldots, d-1, d+1, \ldots, k-1)$

or

(ii) $A_{d}(z)$ is transcendental with $\sigma\left(A_{d}\right)=0$ and $A_{j}(z)(j \neq d)$ are polynomials.

Then every solution $f(z)(\equiv 0)$ of $(1.1)$ is either a polynomial with $\operatorname{deg} f \leq d-1$, or an infinite order entire function. Furthermore, if among $A_{d-1}(z), \ldots, A_{0}(z)$ there exist and only exist 
$A_{m_{1}}(z), \ldots, A_{m_{s}}(z)\left(d-1 \geq m_{1}>m_{2}>\cdots>m_{s} \geq 0\right)$ being transcendental, and $\sigma\left(A_{m_{j}}\right)(j=$ $1, \ldots, s)$ are unequal to each other or $s=1$, and if (1) $m_{s}=0$, or (2) $m_{s}>0$ and polynomials $A_{m_{s}-1}(z), \ldots, A_{0}(z)$ satisfy that $\operatorname{deg} A_{j}-j\left(j=m_{s}-1, \ldots, 0\right)$ are unequal to each other or $m_{s}=1$ and $A_{0}(z) \not \equiv 0$, then every solution $f(z)(\not \equiv 0)$ of $(1.1)$ satisfies $\sigma(f)=\infty$.

Theorems A and B give the properties of solutions of (1.1) when there is exactly one coefficient that has the maximal order. Thus, a natural question arises: how about the properties of solutions of (1.1) when there is more than one coefficient having the maximal order? In this paper, we proceed in this way.

Now, we turn to consider the homogeneous linear differential equation

$$
A_{k}(z) f^{(k)}+A_{k-1}(z) f^{(k-1)}+\cdots+A_{1}(z) f^{\prime}+A_{0}(z) f=0,
$$

where $k \geq 2, A_{k}(z) A_{0}(z) \not \equiv 0$, and $A_{j}(z), j=0,1, \ldots, k$, are entire functions. Hamani and Belaidi in [14] and He et al. in [15] investigated (1.2) and obtained the properties of the iterated order of solutions of (1.2) when there exists one $A_{s}(z)(s \in\{0,1, \ldots, k-1\})$ having the maximal iterated order. When $A_{k}(z)$ has the order larger than the others or $A_{k}(z)$ is transcendental while the others are polynomials, by dividing $(1.2)$ by $A_{k}(z)$, we find that it is just the case when all coefficients of (1.1) are meromorphic and have the same order $\sigma\left(A_{k}\right)$. For this case, we obtain the following results.

Theorem 1.1 Suppose that $k \geq 2, A_{j}(z), j=0,1, \ldots, k$, are entire functions satisfying $A_{k}(z) A_{0}(z) \not \equiv 0$ and $\sigma\left(A_{j}\right)<\sigma\left(A_{k}\right)<\infty, j=0,1, \ldots, k-1$. Suppose that $A_{k}(z)=\sum_{n=0}^{\infty} c_{\lambda_{n}} z^{\lambda_{n}}$ and the sequence of exponents $\left\{\lambda_{n}\right\}$ satisfies the Fabry gap condition

$$
\frac{\lambda_{n}}{n} \rightarrow \infty \quad(n \rightarrow \infty)
$$

Then every rational solution $f(z)$ of $(1.2)$ is a polynomial with $\operatorname{deg} f \leq k-1$, and every transcendental meromorphic solution $f(z)$ of (1.2), whose poles are of uniformly bounded multiplicities such that $\lambda\left(\frac{1}{f}\right)<\mu(f)$, satisfies

$$
\bar{\lambda}(f-\varphi)=\lambda(f-\varphi)=\sigma(f)=\infty, \quad \bar{\lambda}_{2}(f-\varphi)=\lambda_{2}(f-\varphi)=\sigma_{2}(f)=\sigma\left(A_{k}\right),
$$

where $\varphi(z)$ is a finite order meromorphic function and does not solve (1.2).

Remark 1.1 Suppose that $A_{k}(z)=\sum_{n=0}^{\infty} c_{\lambda_{n}} z^{\lambda_{n}}$ is an entire function, and the sequence of exponents $\left\{\lambda_{n}\right\}$ satisfies Fabry gap condition (1.3), then the series $\sum_{n=0}^{\infty} c_{\lambda_{n}} z^{\lambda_{n}}$ is called a Fabry gap series. It follows by [16] that if $A_{k}(z)$ is a Fabry gap series, then it has no deficient values. In particular, zero is not a deficient value of $A_{k}(z)$, then the solutions of (1.2) are meromorphic in general.

Theorem 1.2 Suppose that $k \geq 2, A_{j}(z), j=0,1, \ldots, k$, are entire functions satisfying $A_{k}(z) A_{0}(z) \not \equiv 0$ and

(i) $\sigma\left(A_{j}\right)<\sigma\left(A_{k}\right)<\frac{1}{2}, j=0,1, \ldots, k-1$

\section{or}

(ii) $A_{k}(z)$ is transcendental with $\sigma\left(A_{k}\right)=0$, and $A_{j}(z), j=0,1, \ldots, k-1$, are polynomials. 
Then every rational solution $f(z)$ of $(1.2)$ is a polynomial with $\operatorname{deg} f \leq k-1$, and every transcendental meromorphic solution $f(z)$ of $(1.2)$, whose poles are of uniformly bounded multiplicities such that $\lambda\left(\frac{1}{f}\right)<\mu(f)$, satisfies

$$
\bar{\lambda}(f-\varphi)=\lambda(f-\varphi)=\sigma(f)=\infty, \quad \bar{\lambda}_{2}(f-\varphi)=\lambda_{2}(f-\varphi)=\sigma_{2}(f)=\sigma\left(A_{k}\right),
$$

where $\varphi(z)$ is a finite order meromorphic function and does not solve (1.2).

Next, we consider the non-homogeneous linear differential equation

$$
A_{k}(z) f^{(k)}+A_{k-1}(z) f^{(k-1)}+\cdots+A_{1}(z) f^{\prime}+A_{0}(z) f=F(z)
$$

where $k \geq 2, A_{j}(z), j=0,1, \ldots, k, F(z)$ are entire functions, $A_{k}(z) A_{0}(z) F(z) \not \equiv 0$ and $A_{j}(z)$, $j=0,1, \ldots, k$, satisfy the hypotheses of Theorem 1.1 or 1.2 .

Theorem 1.3 Suppose that $A_{j}(z), j=0,1, \ldots, k$, satisfy the hypotheses of Theorem 1.1 or 1.2, $F(z)(\not \equiv 0)$ is an entire function of finite order.

(i) If $\sigma(F)<\sigma\left(A_{k}\right)$ (now $A_{k}(z)$ does not satisfy (ii) of Theorem 1.2), then every rational solution $f(z)$ of $(1.4)$ is a polynomial with $\operatorname{deg} f \leq k-1$, and every transcendental meromorphic solution $f(z)$ of $(1.4)$, whose poles are of uniformly bounded multiplicities such that $\lambda\left(\frac{1}{f}\right)<\mu(f)$, satisfies

$$
\bar{\lambda}(f-\varphi)=\lambda(f-\varphi)=\sigma(f)=\infty, \quad \bar{\lambda}_{2}(f-\varphi)=\lambda_{2}(f-\varphi)=\sigma_{2}(f)=\sigma\left(A_{k}\right),
$$

where $\varphi(z)$ is a finite order meromorphic function and does not solve (1.4).

(ii) If $\sigma(F)>\sigma\left(A_{k}\right)$, then every infinite order meromorphic solution $f(z)$ of (1.4) satisfies

$$
\bar{\lambda}(f-\varphi)=\lambda(f-\varphi)=\sigma(f)=\infty
$$

where $\varphi(z)$ is a finite order meromorphic function and does not solve (1.4). And every finite order meromorphic solution $f_{0}(z)$ satisfies

$$
\sigma(F) \leq \sigma\left(f_{0}\right) \leq \max \left\{\sigma(F), \bar{\lambda}\left(f_{0}\right)\right\}
$$

For the case of entire solutions, we can deduce the following Corollary 1.1 easily.

Corollary 1.1 Under the hypotheses of Theorem 1.1 or 1.2 or 1.3 , the same conclusions hold for every entire solution of (1.2) or (1.4).

\section{Preliminary lemmas}

Lemma 2.1 (see [17]) Let $f(z)$ be a transcendental meromorphic function of finite order, $\Gamma=\left\{\left(k_{1}, j_{1}\right), \ldots,\left(k_{m}, j_{m}\right)\right\}$ be a finite set of distinct pairs of integers which satisfy $k_{i}>j_{i} \geq 0$ for $i=1, \ldots, m$, and let $\varepsilon>0$ be a given constant. Then there exists a set $E \subset(1,+\infty)$ that has finite logarithmic measure such that for all $z$ satisfying $|z| \notin E \cup[0,1]$ and for all $(k, j) \in \Gamma$, we have

$$
\left|\frac{f^{(k)}(z)}{f^{(j)}(z)}\right| \leq|z|^{(k-j)(\sigma(f)-1+\varepsilon)}
$$


Lemma 2.2 (see [6]) Let $f(z)=\frac{g(z)}{d(z)}$ be a meromorphic function, where $g(z), d(z)$ are entire functions of finite iterated order satisfying $i(f)=p \in \mathbb{N}, \mu_{p}(g)=\mu_{p}(f) \leq \sigma_{p}(g)=\sigma_{p}(f), i(d)<$ p or $\sigma_{p}(d)<\mu_{p}(f)$. Let $z$ be a point with $|z|=r$ at which $|g(z)|=M(r, g)$, and let $v_{g}(r)$ denote the central index of $g(z)$, then the estimation

$$
\frac{f^{(k)}(z)}{f(z)}=\left(\frac{\nu_{g}(r)}{z}\right)^{k}(1+o(1)), \quad k \in \mathbb{N}
$$

holds for all $|z|=r$ outside a set $E$ of $r$ of finite logarithmic measure.

Lemma 2.3 Let $f(z)$ satisfy the hypotheses of Lemma 2.2, then there exists a set $E \subset(1,+\infty)$ having finite logarithmic measure such that for all z satisfying $|z|=r \notin E \cup[0,1]$ and $|g(z)|=$ $M(r, g)$, we have

$$
\left|\frac{f(z)}{f^{(k)}(z)}\right| \leq 2 r^{k}, \quad k \in \mathbb{N} .
$$

Proof It follows by Lemma 2.2 that

$$
\frac{f^{(k)}(z)}{f(z)}=\left(\frac{v_{g}(r)}{z}\right)^{k}(1+o(1)),
$$

where $|z|=r \notin E \cup[0,1], E \subset(1,+\infty)$ is of finite logarithmic measure and $|g(z)|=M(r, g)$. Since $g(z)$ is transcendental, $v_{g}(r) \rightarrow \infty(r \rightarrow \infty)$. Hence, when $z$ satisfies $|z|=r \notin E \cup[0,1]$ and $|g(z)|=M(r, g)$, we get Lemma 2.3 .

Lemma 2.4 (see $[13,16])$ Let $A(z)=\sum_{n=0}^{\infty} c_{\lambda_{n}} z^{\lambda_{n}}$ be an entire function of finite order and the sequence of exponents $\left\{\lambda_{n}\right\}$ satisfy (1.3), and $f(z)$ be an entire function satisfying $\sigma(f)=$ $\sigma \in(0,+\infty)$. Then, for any given $\varepsilon(0<\varepsilon<\sigma)$, there exists a set $H \subset(1,+\infty)$ satisfying $\overline{\log \operatorname{dens}} H \geq \eta$, where $\eta \in(0,1)$ is a constant, such that for all $z$ satisfying $|z|=r \in H$, one has

$$
\log M(r, f)>r^{\sigma-\varepsilon}, \quad \log L(r, A)>(1-\eta) \log M(r, A)
$$

where $L(r, A)=\min _{|z|=r}|A(z)|, M(r, A)=\max _{|z|=r}|A(z)|, M(r, f)=\max _{|z|=r}|f(z)|$.

We may deduce the following Remark 2.1 from Lemma 2.4 immediately.

Remark 2.1 Let $A(z)=\sum_{n=0}^{\infty} c_{\lambda_{n}} z^{\lambda_{n}}$ be an entire function satisfying $\sigma(A)=\sigma \in(0,+\infty)$, and let the sequence of exponents $\left\{\lambda_{n}\right\}$ satisfy (1.3). Then, for any given $\varepsilon(0<2 \varepsilon<\sigma)$, there exists a set $H \subset(1,+\infty)$ satisfying $\overline{\log \operatorname{dens}} H \geq \eta$, where $\eta \in(0,1)$ is a constant such that for all $z$ satisfying $|z|=r \in H$, one has

$$
|A(z)|>[M(r, A)]^{1-\eta}>\exp \left\{(1-\eta) r^{\sigma-\varepsilon}\right\}>\exp \left\{r^{\sigma-2 \varepsilon}\right\} .
$$

Lemma 2.5 (see [6]) Let $A_{j}(z), j=0,1, \ldots, k-1, F(z)(\not \equiv 0)$ be meromorphic functions, and $f(z)$ be a meromorphic solution of

$$
f^{(k)}+A_{k-1}(z) f^{(k-1)}+\cdots+A_{1}(z) f^{\prime}+A_{0}(z) f=F(z)
$$


satisfying one of the following conditions:

(i) $\max \left\{i(F)=q, i\left(A_{j}\right)(j=0,1, \ldots, k-1)\right\}<i(f)=p+1(0<p<\infty)$,

(ii) $b=\max \left\{\sigma_{p+1}(F), \sigma_{p+1}\left(A_{j}\right)(j=0,1, \ldots, k-1)\right\}<\sigma_{p+1}(f)=\sigma$.

Then $\bar{\lambda}_{p+1}(f)=\lambda_{p+1}(f)=\sigma_{p+1}(f)=\sigma$.

Lemma 2.6 (see [12]) Suppose that $k \geq 2, A_{j}(z), j=0,1, \ldots, k-1$, are meromorphic functions, $\sigma=\max \left\{\sigma\left(A_{j}\right), j=0,1, \ldots, k-1\right\}$. If $f(z)$ is a transcendental meromorphic solution of (1.1) and all poles of $f(z)$ are of uniformly bounded multiplicity, then we have $\sigma_{2}(f) \leq \sigma$.

Lemma 2.7 (see [17]) Let $f(z)$ be a transcendental meromorphic function, $\Gamma=\left\{\left(k_{1}, j_{1}\right), \ldots\right.$, $\left.\left(k_{m}, j_{m}\right)\right\}$ be a finite set of distinct pairs of integers which satisfy $k_{i}>j_{i} \geq 0$ for $i=1, \ldots, m$, and $\alpha>1$ be a given constant. Then there exists a set $E \subset(1,+\infty)$ that has finite logarithmic measure, and exists a constant $C>0$, that depends only on $\alpha$ and $\Gamma$, such that for all $z$ satisfying $|z| \notin E \cup[0,1]$ and for all $(k, j) \in \Gamma$, we have

$$
\left|\frac{f^{(k)}(z)}{f^{(j)}(z)}\right| \leq C\left[\frac{T(\alpha r, f)}{r} \log ^{\alpha} r \log T(\alpha r, f)\right]^{k-j}
$$

Lemma 2.8 (see [18]) Let $f(z)$ be an entire function of order $\sigma(f)=\sigma<\frac{1}{2}$ and denote $A(r)=$ $\inf _{|z|=r} \log |f(z)|, B(r)=\sup _{|z|=r} \log |f(z)|$.If $\sigma<\alpha<\frac{1}{2}$, then

$$
\underline{\log \operatorname{dens}}\{r: A(r)>(\cos \pi \alpha) B(r)\} \geq 1-\frac{\sigma}{\alpha} .
$$

Lemma 2.9 (see [19]) Let $f(z)$ be an entire function with $\mu(f)=\mu<\frac{1}{2}$ and $\mu<\sigma=\sigma(f)$. If $\mu \leq \delta<\min \left\{\sigma, \frac{1}{2}\right\}$ and $\delta<\alpha<\frac{1}{2}$, then

$$
\overline{\log \operatorname{dens}}\left\{r: A(r)>(\cos \pi \alpha) B(r)>r^{\delta}\right\}>C(\sigma, \delta, \alpha),
$$

where $C(\sigma, \delta, \alpha)$ is a positive constant depending only on $\sigma, \delta, \alpha$.

Lemma 2.10 (see [11]) Let $f(z)$ be a meromorphic function with $\sigma(f)=\beta<\infty$. Then, for any $\varepsilon>0$, there exists a set $E \subset(1,+\infty)$ with $m E<\infty$ such that for all $z$ with $|z|=r \notin$ $E \cup[0,1], r \rightarrow \infty$, we have

$$
\exp \left\{-r^{\beta+\varepsilon}\right\} \leq|f(z)| \leq \exp \left\{r^{\beta+\varepsilon}\right\}
$$

Lemma 2.11 (see [20]) Let $g:[0,+\infty) \rightarrow \mathbb{R}$ and $h:[0,+\infty) \rightarrow \mathbb{R}$ be monotone nondecreasing functions such that $g(r) \leq h(r)$ for all $r \notin E \cup[0,1]$, where $E \subset(1,+\infty)$ is a set of finite logarithmic measure. Let $\alpha>1$ be a given constant. Then there exists $r_{0}=r_{0}(\alpha)>0$ such that $g(r) \leq h(\alpha r)$ for all $r>r_{0}$.

Lemma 2.12 (see [6]) Let $f(z)$ be an entire function of finite iterated order with $i(f)=p+1$, $p \in \mathbb{N}$, and $v_{f}(r)$ be the central index of $f(z)$. Then

$$
\varlimsup_{r \rightarrow \infty} \frac{\log _{p+1} v_{f}(r)}{\log r}=\sigma_{p+1}(f) .
$$




\section{Proofs of Theorems 1.1-1.3}

Proof of Theorem 1.1 Suppose that $f(z)$ is a rational solution of (1.2). Since $\sigma\left(A_{k}\right)>$ $\max \left\{\sigma\left(A_{j}\right), j=0,1, \ldots, k-1\right\}$, it is clear that $f(z)$ is a polynomial with $\operatorname{deg} f \leq k-1$.

Now, suppose that $f(z)$ is a transcendental meromorphic solution of (1.2), whose poles are of uniformly bounded multiplicities such that $\lambda\left(\frac{1}{f}\right)<\mu(f)$. And we suppose on the contrary that $\sigma(f)<\infty$. By Lemma 2.1, there exists a set $E_{1} \subset(1,+\infty)$ of finite logarithmic measure such that for all $z$ satisfying $|z|=r \notin[0,1] \cup E_{1}$, we have

$$
\left|\frac{f^{(j)}(z)}{f(z)}\right| \leq r^{k \cdot \sigma(f)}, \quad j=1, \ldots, k-1
$$

Since $\lambda\left(\frac{1}{f}\right)<\mu(f)$, by Hadamard's factorization theorem, we may denote $f(z)$ as $f(z)=\frac{g(z)}{d(z)}$, where $g(z)$ and $d(z)$ are entire functions and $d(z)$ is the canonical product formed by all poles of $f(z)$ such that $\sigma(d)=\lambda\left(\frac{1}{f}\right)<\mu(f)=\mu(g) \leq \sigma(g)=\sigma(f)<\infty$. Then, by Lemma 2.3, there exists a set $E_{2} \subset(1,+\infty)$ of finite logarithmic measure such that for all $z$ satisfying $|z|=r \notin[0,1] \cup E_{2}$ and $|g(z)|=M(r, g)$, we have

$$
\left|\frac{f(z)}{f^{(k)}(z)}\right| \leq 2 r^{k}
$$

Set $\sigma=\sigma\left(A_{k}\right)$ and $\beta=\max \left\{\sigma\left(A_{j}\right), j=0,1, \ldots, k-1\right\}$. Since $\beta<\sigma$, for any given $\varepsilon(0<\varepsilon<$ $\left.\frac{\sigma-\beta}{2}\right)$ and sufficiently large $r=|z|$, we have

$$
\left|A_{j}(z)\right| \leq M\left(r, A_{j}\right) \leq \exp \left\{r^{\beta+\varepsilon}\right\}, \quad j=0,1, \ldots, k-1 .
$$

By Remark 2.1, for the above $\varepsilon$, there exists a set $H_{1} \subset(1,+\infty)$ of infinite logarithmic measure such that for all $z$ satisfying $|z|=r \in H_{1}$, we have

$$
\left|A_{k}(z)\right| \geq \exp \left\{r^{\sigma-\varepsilon}\right\}
$$

And from (1.2) we can obtain that

$$
-A_{k}(z)=\frac{f}{f^{(k)}}\left(A_{k-1}(z) \frac{f^{(k-1)}}{f}+\cdots+A_{1}(z) \frac{f^{\prime}}{f}+A_{0}(z)\right) .
$$

Combining (3.1)-(3.5), we have

$$
\exp \left\{r^{\sigma-\varepsilon}\right\} \leq 2 k r^{k} \cdot \exp \left\{r^{\beta+\varepsilon}\right\} \cdot r^{k \cdot \sigma(f)}, \quad r \in H_{1} \backslash\left(E_{1} \cup E_{2} \cup[0,1]\right) .
$$

Noting that $0<\varepsilon<\frac{\sigma-\beta}{2}$, we can see that (3.6) is a contradiction. Therefore, every transcendental meromorphic solution $f(z)$ of (1.2), whose poles are of uniformly bounded multiplicities such that $\lambda\left(\frac{1}{f}\right)<\mu(f)$, satisfies $\sigma(f)=\infty$.

Next, we prove $\bar{\lambda}(f-\varphi)=\lambda(f-\varphi)=\sigma(f)=\infty$. Set $g(z)=f(z)-\varphi(z)$, then $g(z)$ solves the equation

$$
\begin{aligned}
g^{(k)} & +\frac{A_{k-1}(z)}{A_{k}(z)} g^{(k-1)}+\cdots+\frac{A_{1}(z)}{A_{k}(z)} g^{\prime}+\frac{A_{0}(z)}{A_{k}(z)} g \\
& =-\varphi^{(k)}-\frac{A_{k-1}(z)}{A_{k}(z)} \varphi^{(k-1)}-\cdots-\frac{A_{1}(z)}{A_{k}(z)} \varphi^{\prime}-\frac{A_{0}(z)}{A_{k}(z)} \varphi
\end{aligned}
$$


and satisfies $\sigma(g)=\sigma(f)=\infty$. Since $\varphi(z)$ does not solve (1.2), we have that

$$
-\varphi^{(k)}-\frac{A_{k-1}(z)}{A_{k}(z)} \varphi^{(k-1)}-\cdots-\frac{A_{1}(z)}{A_{k}(z)} \varphi^{\prime}-\frac{A_{0}(z)}{A_{k}(z)} \varphi \not \equiv 0 .
$$

Then, by Lemma 2.5 and $\sigma(\varphi)<\infty$, we have

$$
\bar{\lambda}(g)=\lambda(g)=\sigma(g)=\infty,
$$

that is,

$$
\bar{\lambda}(f-\varphi)=\lambda(f-\varphi)=\sigma(f)=\infty .
$$

In the end, we prove $\bar{\lambda}_{2}(f-\varphi)=\lambda_{2}(f-\varphi)=\sigma_{2}(f)=\sigma$. By Lemma 2.6, every transcendental meromorphic solution $f(z)$, whose poles are of uniformly bounded multiplicities, of the equation

$$
f^{(k)}+\frac{A_{k-1}(z)}{A_{k}(z)} f^{(k-1)}+\cdots+\frac{A_{1}(z)}{A_{k}(z)} f^{\prime}+\frac{A_{0}(z)}{A_{k}(z)} f=0
$$

satisfies $\sigma_{2}(f) \leq \max \left\{\sigma\left(\frac{A_{j}}{A_{k}}\right), j=0,1, \ldots, k-1\right\}=\sigma\left(A_{k}\right)=\sigma$. On the other hand, by Lemma 2.7, there exist a set $E_{3} \subset(1,+\infty)$ that has finite logarithmic measure and a constant $B>0$ such that for all $z$ satisfying $|z|=r \notin[0,1] \cup E_{3}$, we have

$$
\left|\frac{f^{(j)}(z)}{f(z)}\right| \leq B(T(2 r, f))^{2 k}, \quad j=1, \ldots, k-1
$$

Combining (3.2)-(3.5) with (3.8), we have

$$
\exp \left\{r^{\sigma-\varepsilon}\right\} \leq 2 k r^{k} \cdot \exp \left\{r^{\beta+\varepsilon}\right\} \cdot B(T(2 r, f))^{2 k}, \quad r \in H_{1} \backslash\left(E_{2} \cup E_{3} \cup[0,1]\right),
$$

which implies $\sigma_{2}(f) \geq \sigma-\varepsilon$. Since $\varepsilon\left(0<\varepsilon<\frac{\sigma-\beta}{2}\right)$ is arbitrary, $\sigma_{2}(f) \geq \sigma=\sigma\left(A_{k}\right)$ holds. Therefore, we have $\sigma_{2}(f)=\sigma=\sigma\left(A_{k}\right)$.

By using a similar method as above and Lemma 2.5, we can obtain that

$$
\bar{\lambda}_{2}(f-\varphi)=\lambda_{2}(f-\varphi)=\sigma_{2}(f)=\sigma\left(A_{k}\right) .
$$

Proof of Theorem 1.2 (i) Suppose that $f(z)$ is a rational solution of (1.2). Since $\sigma\left(A_{k}\right)>$ $\max \left\{\sigma\left(A_{j}\right), j=0,1, \ldots, k-1\right\}$, it is clear that $f(z)$ is a polynomial with $\operatorname{deg} f \leq k-1$.

Now, suppose that $f(z)$ is a transcendental meromorphic solution of (1.2), whose poles are of uniformly bounded multiplicities such that $\lambda\left(\frac{1}{f}\right)<\mu(f)$. And we suppose on the contrary that $\sigma(f)<\infty$. Since $\lambda\left(\frac{1}{f}\right)<\mu(f)$, by Hadamard's factorization theorem, we may denote $f(z)$ as $f(z)=\frac{g(z)}{d(z)}$, where $g(z)$ and $d(z)$ are entire functions and $d(z)$ is the canonical product formed by all poles of $f(z)$ such that $\sigma(d)=\lambda\left(\frac{1}{f}\right)<\mu(f)=\mu(g) \leq \sigma(g)=\sigma(f)<\infty$. Then (3.1) and (3.2) hold for all $z$ satisfying $|z|=r \notin[0,1] \cup E_{1} \cup E_{2}$ and $|g(z)|=M(r, g)$. Now, we choose two constants $\tau, \gamma$ such that for $j=0,1, \ldots, k-1$,

$$
\sigma\left(A_{j}\right)<\tau<\gamma<\sigma\left(A_{k}\right)
$$


Since $\sigma=\sigma\left(A_{k}\right)<\frac{1}{2}$, by Lemmas 2.8 and 2.9, there exists a set $H_{2} \subset(1,+\infty)$ of infinite logarithmic measure such that for all $z$ satisfying $|z|=r \in H_{2}$, we have

$$
\log \left|A_{k}(z)\right|>r^{\gamma}
$$

Since $\sigma\left(A_{j}\right)<\tau, j=0,1, \ldots, k-1$, we have, for sufficiently large $r=|z|$,

$$
\left|A_{j}(z)\right| \leq \exp \left\{r^{\tau}\right\}, \quad j=0,1, \ldots, k-1 .
$$

Then (3.1), (3.2), (3.5), (3.9), (3.10) imply that

$$
\exp \left\{r^{\gamma}\right\} \leq 2 k r^{k} \cdot \exp \left\{r^{\tau}\right\} \cdot r^{k \cdot \sigma(f)}, \quad r \in H_{2} \backslash\left(E_{1} \cup E_{2} \cup[0,1]\right)
$$

which is a contradiction. Therefore, every transcendental meromorphic solution $f(z)$ of (1.2), whose poles are of uniformly bounded multiplicities such that $\lambda\left(\frac{1}{f}\right)<\mu(f)$, satisfies $\sigma(f)=\infty$.

By Lemma 2.6, every transcendental meromorphic solution $f(z)$, whose poles are of uniformly bounded multiplicities, of equation (3.7) satisfies $\sigma_{2}(f) \leq \max \left\{\sigma\left(\frac{A_{j}}{A_{k}}\right), j=0,1, \ldots, k-\right.$ $1\}=\sigma\left(A_{k}\right)$. On the other hand, combining (3.2), (3.5) with (3.8)-(3.10), we have

$$
\exp \left\{r^{\gamma}\right\} \leq 2 k r^{k} \cdot \exp \left\{r^{\tau}\right\} \cdot B(T(2 r, f))^{2 k}, \quad r \in H_{2} \backslash\left(E_{2} \cup E_{3} \cup[0,1]\right)
$$

which implies $\sigma_{2}(f) \geq \gamma$. Letting $\gamma \rightarrow \sigma\left(A_{k}\right)$, we have $\sigma_{2}(f) \geq \sigma\left(A_{k}\right)$. Therefore, we have $\sigma_{2}(f)=\sigma\left(A_{k}\right)$.

By using a similar method as the one in the proof of Theorem 1.1, we can prove

$$
\bar{\lambda}(f-\varphi)=\lambda(f-\varphi)=\sigma(f)=\infty, \quad \bar{\lambda}_{2}(f-\varphi)=\lambda_{2}(f-\varphi)=\sigma_{2}(f)=\sigma\left(A_{k}\right) .
$$

(ii) Suppose that $f(z)$ is a rational solution of (1.2). Since $A_{k}(z)$ is transcendental and $A_{j}(z), j=0,1, \ldots, k-1$, are polynomials, we can easily obtain that $f(z)$ is a polynomial with $\operatorname{deg} f \leq k-1$.

Now, suppose that $f(z)$ is a transcendental meromorphic solution of (1.2), whose poles are of uniformly bounded multiplicities such that $\lambda\left(\frac{1}{f}\right)<\mu(f)$. And we suppose on the contrary that $\sigma(f)<\infty$. Since $\lambda\left(\frac{1}{f}\right)<\mu(f)$, by Hadamard's factorization theorem, we may denote $f(z)$ as $f(z)=\frac{g(z)}{d(z)}$, where $g(z)$ and $d(z)$ are entire functions and $d(z)$ is the canonical product formed by all poles of $f(z)$ such that $\sigma(d)=\lambda\left(\frac{1}{f}\right)<\mu(f)=\mu(g) \leq \sigma(g)=\sigma(f)<\infty$. Then (3.1) and (3.2) hold for all $z$ satisfying $|z|=r \notin[0,1] \cup E_{1} \cup E_{2}$ and $|g(z)|=M(r, g)$. Since $A_{j}(z), j=0,1, \ldots, k-1$, are polynomials, there exists $M>0$ such that

$$
\left|A_{j}(z)\right| \leq r^{M}, \quad j=0,1, \ldots, k-1
$$

Since $A_{k}(z)$ is transcendental, by Lemma 2.8 , there exists a set $H_{3} \subset(1,+\infty)$ of infinite logarithmic measure such that for all $z$ satisfying $|z|=r \in H_{3}$, we have

$$
\frac{\min \left\{\log \left|A_{k}(z)\right|:|z|=r\right\}}{\log r} \rightarrow \infty
$$


Hence,

$$
\left|A_{k}(z)\right| \geq r^{k(\sigma(f)+1)+2 M}, \quad|z|=r \in H_{3}
$$

Then (3.1), (3.2), (3.5), (3.11) and (3.12) imply that

$$
r^{k(\sigma(f)+1)+2 M} \leq 2 k r^{k} \cdot r^{M} \cdot r^{k \cdot \sigma(f)}=2 k r^{k(\sigma(f)+1)+M}, \quad r \in H_{3} \backslash\left(E_{1} \cup E_{2} \cup[0,1]\right),
$$

which is a contradiction. Therefore, every transcendental meromorphic solution $f(z)$ of (1.2), whose poles are of uniformly bounded multiplicities such that $\lambda\left(\frac{1}{f}\right)<\mu(f)$, satisfies $\sigma(f)=\infty$.

By using a similar method as the one in the proof of Theorem 1.1, we can obtain that

$$
\bar{\lambda}(f-\varphi)=\lambda(f-\varphi)=\sigma(f)=\infty .
$$

Now, (3.7) together with Lemma 2.6 implies that

$$
\sigma_{2}(f) \leq \max \left\{\sigma\left(A_{j}\right), j=0,1, \ldots, k\right\}=0
$$

that is,

$$
\bar{\lambda}_{2}(f-\varphi)=\lambda_{2}(f-\varphi)=\sigma_{2}(f)=\sigma\left(A_{k}\right)=0 .
$$

Proof of Theorem 1.3 We prove only the case under the hypotheses of Theorem 1.1, and the case under the hypotheses of Theorem 1.2 can be proved similarly. So, we omit the proof of the second case.

(i) Suppose that $f(z)$ is a rational solution of (1.4). Since $\sigma\left(A_{k}\right)>\max \left\{\sigma\left(A_{j}\right), j=0,1, \ldots, k-\right.$ $1, \sigma(F)\}$, it is clear that $f(z)$ is a polynomial with $\operatorname{deg} f \leq k-1$.

Now, suppose that $f(z)$ is a transcendental meromorphic solution of (1.4), whose poles are of uniformly bounded multiplicities such that $\lambda\left(\frac{1}{f}\right)<\mu(f)$. And we suppose on the contrary that $\sigma(f)<\infty$. Since $\lambda\left(\frac{1}{f}\right)<\mu(f)$, by Hadamard's factorization theorem, we may denote $f(z)$ as $f(z)=\frac{g(z)}{d(z)}$, where $g(z)$ and $d(z)$ are entire functions and $d(z)$ is the canonical product formed by all poles of $f(z)$ such that $\sigma(d)=\lambda\left(\frac{1}{f}\right)<\mu(f)=\mu(g) \leq \sigma(g)=\sigma(f)<\infty$. Then (3.1) and (3.2) hold for all $z$ satisfying $|z|=r \notin[0,1] \cup E_{1} \cup E_{2}$ and $|g(z)|=M(r, g)$. Set $\sigma=\sigma\left(A_{k}\right)$ and $\delta=\max \left\{\sigma\left(A_{j}\right), j=0,1, \ldots, k-1, \sigma(F)\right\}$. Since $\delta<\sigma$, for any given $\varepsilon(0<\varepsilon<$ $\left.\min \left\{\frac{\sigma-\delta}{2}, \frac{\mu(f)-\lambda\left(\frac{1}{f}\right)}{2}\right\}\right)$ and sufficiently large $r=|z|$, we have

$$
|F(z)| \leq \exp \left\{r^{\delta+\varepsilon}\right\}, \quad\left|A_{j}(z)\right| \leq \exp \left\{r^{\delta+\varepsilon}\right\}, \quad j=0,1, \ldots, k-1 .
$$

Moreover, (3.4) holds for all $z$ satisfying $|z|=r \in H_{1}$. Since $\sigma(d)=\lambda\left(\frac{1}{f}\right)<\mu(f)=\mu(g)$, for the above $\varepsilon$ and sufficiently large $z$ satisfying $|z|=r$ and $|g(z)|=M(r, g)$, we have that

$$
\left|\frac{1}{f(z)}\right|=\left|\frac{d(z)}{M(r, g)}\right| \leq\left|\frac{\exp \left\{r^{\lambda\left(\frac{1}{f}\right)+\varepsilon}\right\}}{\exp \left\{r^{\mu(f)-\varepsilon}\right\}}\right| \leq 1 .
$$

In addition, (1.4) implies

$$
-A_{k}(z)=\frac{f}{f^{(k)}}\left(A_{k-1}(z) \frac{f^{(k-1)}}{f}+\cdots+A_{1}(z) \frac{f^{\prime}}{f}+A_{0}(z)-F(z) \frac{1}{f}\right) .
$$


Then (3.1), (3.2), (3.4), (3.13)-(3.15) imply that

$$
\exp \left\{r^{\sigma-\varepsilon}\right\} \leq 2 r^{k} \cdot r^{k \cdot \sigma(f)} \cdot(k+1) \exp \left\{r^{\delta+\varepsilon}\right\}, \quad r \in H_{1} \backslash\left(E_{1} \cup E_{2} \cup[0,1]\right)
$$

Since $0<\varepsilon<\frac{\sigma-\delta}{2}$, (3.16) is a contradiction. Therefore, every transcendental meromorphic solution $f(z)$ of (1.4), whose poles are of uniformly bounded multiplicities such that $\lambda\left(\frac{1}{f}\right)<\mu(f)$, satisfies $\sigma(f)=\infty$.

Set $g(z)=f(z)-\varphi(z)$, then $g(z)$ solves the equation

$$
\begin{aligned}
g^{(k)} & +\frac{A_{k-1}(z)}{A_{k}(z)} g^{(k-1)}+\cdots+\frac{A_{1}(z)}{A_{k}(z)} g^{\prime}+\frac{A_{0}(z)}{A_{k}(z)} g \\
= & \frac{F(z)}{A_{k}(z)}-\varphi^{(k)}-\frac{A_{k-1}(z)}{A_{k}(z)} \varphi^{(k-1)}-\cdots-\frac{A_{1}(z)}{A_{k}(z)} \varphi^{\prime}-\frac{A_{0}(z)}{A_{k}(z)} \varphi
\end{aligned}
$$

and satisfies $\sigma(g)=\sigma(f)=\infty$. Since $\varphi(z)$ does not solve (1.4), we have that

$$
\frac{F(z)}{A_{k}(z)}-\varphi^{(k)}-\frac{A_{k-1}(z)}{A_{k}(z)} \varphi^{(k-1)}-\cdots-\frac{A_{1}(z)}{A_{k}(z)} \varphi^{\prime}-\frac{A_{0}(z)}{A_{k}(z)} \varphi \not \equiv 0
$$

By Lemma 2.5, we have

$$
\bar{\lambda}(g)=\lambda(g)=\sigma(g)=\infty,
$$

that is,

$$
\bar{\lambda}(f-\varphi)=\lambda(f-\varphi)=\sigma(f)=\infty .
$$

By using (3.13)-(3.15) instead of (3.3) and (3.5) and using a similar method as the one in the proof of Theorem 1.1, we can prove $\sigma_{2}(f) \geq \sigma\left(A_{k}\right)$. (As for the case under the hypotheses of Theorem 1.2(i), combining (3.2), (3.8), (3.9) with (3.13)-(3.15), we have

$$
\exp \left\{r^{\gamma}\right\} \leq 2(k+1) r^{k} \cdot \exp \left\{r^{\delta+\varepsilon}\right\} \cdot B(T(2 r, f))^{2 k}, \quad r \in H_{2} \backslash\left(E_{2} \cup E_{3} \cup[0,1]\right),
$$

which implies $\sigma_{2}(f) \geq \gamma$. Letting $\gamma \rightarrow \sigma\left(A_{k}\right)$, we have $\sigma_{2}(f) \geq \sigma\left(A_{k}\right)$.) Now, we turn to prove that $\sigma_{2}(f) \leq \sigma\left(A_{k}\right)$. Equation (1.4) also implies that

$$
-\frac{f^{(k)}}{f}=\frac{A_{k-1}(z)}{A_{k}(z)} \frac{f^{(k-1)}}{f}+\cdots+\frac{A_{1}(z)}{A_{k}(z)} \frac{f^{\prime}}{f}+\frac{A_{0}(z)}{A_{k}(z)}-\frac{F(z)}{A_{k}(z)} \frac{1}{f} .
$$

Then, by Lemma 2.2 , there exists a set $E_{4} \subset(1,+\infty)$ of finite logarithmic measure such that for any $z$ satisfying $|z|=r \notin E_{4} \cup[0,1]$ and $|g(z)|=M(r, g)$, we have

$$
\begin{aligned}
\left|\frac{v_{g}^{k}(r)}{z^{k}}(1+o(1))\right| \leq & \left|\frac{v_{g}^{k-1}(r)}{z^{k-1}}(1+o(1))\right|\left|\frac{A_{k-1}(z)}{A_{k}(z)}\right|+\cdots+\left|\frac{v_{g}(r)}{z}(1+o(1))\right| \\
& \cdot\left|\frac{A_{1}(z)}{A_{k}(z)}\right|+\left|\frac{A_{0}(z)}{A_{k}(z)}\right|+\left|\frac{F(z)}{A_{k}(z)}\right| \cdot \frac{|d(z)|}{M(r, g)} .
\end{aligned}
$$


Since $\max \left\{\sigma\left(\frac{A_{k-1}}{A_{k}}\right), \ldots, \sigma\left(\frac{A_{0}}{A_{k}}\right), \sigma\left(\frac{F}{A_{k}}\right)\right\}=\sigma\left(A_{k}\right)<\infty$, by Lemma 2.10, for any given $\varepsilon>0$, there exists a set $E_{5} \subset(1,+\infty)$ of finite logarithmic measure such that for any $z$ satisfying $|z|=r \notin E_{5} \cup[0,1]$, we have

$$
\left|\frac{F(z)}{A_{k}(z)}\right| \leq \exp \left\{r^{\sigma\left(A_{k}\right)+\varepsilon}\right\}, \quad\left|\frac{A_{j}(z)}{A_{k}(z)}\right| \leq \exp \left\{r^{\sigma\left(A_{k}\right)+\varepsilon}\right\}, \quad j=0,1, \ldots, k-1 .
$$

Then (3.14), (3.17) and (3.18) imply that for sufficiently large $r \notin E_{4} \cup E_{5} \cup[0,1]$, we have

$$
\frac{1}{2} v_{g}^{k}(r) \leq 2(k+1) r^{k} \cdot v_{g}^{k-1}(r) \cdot \exp \left\{r^{\sigma\left(A_{k}\right)+\varepsilon}\right\}
$$

that is,

$$
\nu_{g}(r) \leq 4(k+1) r^{k} \cdot \exp \left\{r^{\sigma\left(A_{k}\right)+\varepsilon}\right\}
$$

By Lemmas 2.11 and 2.12, we can obtain that $\sigma_{2}(f)=\sigma_{2}(g) \leq \sigma\left(A_{k}\right)+\varepsilon$. Since $\varepsilon(>0)$ is arbitrary, we have $\sigma_{2}(f) \leq \sigma\left(A_{k}\right)$. Therefore, $\sigma_{2}(f)=\sigma\left(A_{k}\right)>0$. And from (1.4), Lemma 2.5 and the fact that

$$
\sigma_{2}(f)=\sigma\left(A_{k}\right)>0=\max \left\{\sigma_{2}\left(\frac{A_{j}}{A_{k}}\right), j=0,1, \ldots, k-1, \sigma_{2}\left(\frac{F}{A_{k}}\right)\right\}
$$

we have

$$
\bar{\lambda}_{2}(f)=\lambda_{2}(f)=\sigma_{2}(f)=\sigma\left(A_{k}\right) .
$$

By using a similar method as above, we can obtain that

$$
\bar{\lambda}_{2}(f-\varphi)=\lambda_{2}(f-\varphi)=\sigma_{2}(f)=\sigma\left(A_{k}\right) .
$$

(ii) Suppose that $f(z)$ is an infinite order meromorphic solution of (1.4). By using a similar method as the one above, we can obtain that

$$
\bar{\lambda}(f-\varphi)=\lambda(f-\varphi)=\sigma(f)=\infty .
$$

For every finite order meromorphic solution $f_{0}(z)$ of (1.4), by a similar reasoning as the one in the proof of Theorem 1 of [10], we easily know that

$$
T\left(r, f_{0}\right) \leq k \bar{N}\left(r, \frac{1}{f_{0}}\right)+(k+1) r^{\sigma(F)+\varepsilon}+O(\log r)
$$

holds for sufficiently large $r$ and any given $\varepsilon>0$. Therefore, we have $\sigma\left(f_{0}\right) \leq \max \{\sigma(F)$, $\left.\bar{\lambda}\left(f_{0}\right)\right\}$. We also have $\sigma(F) \leq \sigma\left(f_{0}\right)$ from (1.4). Hence, we have

$$
\sigma(F) \leq \sigma\left(f_{0}\right) \leq \max \left\{\sigma(F), \bar{\lambda}\left(f_{0}\right)\right\}
$$


Authors' contributions

All authors drafted the manuscript, read and approved the final manuscript.

\section{Acknowledgements}

This work was supported by the National Natural Science Foundation of China $(11301233,11171119)$, the Youth Science Foundation of Education Bureau of Jiangxi Province (GJJ14271) and Sponsored Program for Cultivating Youths of Outstanding Ability in Jiangxi Normal University of China.

Received: 31 July 2014 Accepted: 20 January 2015 Published online: 31 January 2015

\section{References}

1. Hayman, WK: Meromorphic Functions. Clarendon, Oxford (1964)

2. Yang, L: Value Distribution Theory and Its New Research. Science Press, Beijing (1982) (in Chinese)

3. Yang, CC, Yi, HX: Uniqueness Theory of Meromorphic Functions. Mathematics and Its Applications, vol. 557. Kluwer Academic, Dordrecht (2003)

4. $\mathrm{Cao}, \mathrm{TB}, \mathrm{Xu}, \mathrm{JF}$, Chen, ZX: On the meromorphic solutions of linear differential equations on the complex plane. J. Math. Anal. Appl. 364(1), 130-142 (2010)

5. Kinnunen, L: Linear differential equations with solutions of finite iterated order. Southeast Asian Bull. Math. 22(4), 385-405 (1998)

6. Tu, J, Chen, ZX: Growth of solutions of complex differential equations with meromorphic coefficients of finite iterated order. Southeast Asian Bull. Math. 33, 153-164 (2009)

7. Belaïdi, B, Hamouda, S: Growth of solutions of an $n$-th order linear differential equation with entire coefficients. Kodai Math. J. 25, 240-245 (2002)

8. Belaïdi, B, Hamani, K: Order and hyper-order of entire solutions of linear differential equations with entire coefficients. Electron. J. Differ. Equ. 2003, 17 (2003)

9. Chen, ZX, Gao, SA: The complex oscillation theory of certain non-homogeneous linear differential equations with transcendental entire coefficients. J. Math. Anal. Appl. 179, 403-416 (1993)

10. Chen, ZX, Gao, SA: Entire solutions of differential equations with finite order transcendental entire coefficients. Acta Math. Sin. 13(4), 453-464 (1997)

11. Chen, ZX: The growth of solutions of a type of second order differential equations with entire coefficients. Chin. Ann. Math., Ser. A 20(1), 7-14 (1999) (in Chinese)

12. Chen, WJ, Xu, JF: Growth of meromorphic solutions of higher-order linear differential equations. Electron. J. Qual. Theory Differ. Equ. 2009, 1 (2009)

13. Lan, ST, Chen, ZX: On the growth of solutions of higher order differential equation. Acta Math. Appl. Sin. 36(5), 851-861 (2013) (in Chinese)

14. Hamani, K, Belaïdi, B: Growth of solutions of complex linear differential equations with entire coefficients of finite iterated order. Acta Univ. Apulensis 27, 203-216 (2011)

15. He, J, Zheng, XM, Hu, H: Iterated order of meromorphic solutions of certain higher order linear differential equations with meromorphic coefficients of finite iterated order. Acta Univ. Apulensis 33, 145-157 (2013)

16. Hayman, WK, Rossi, JF: Characteristic, maximum modulus and value distribution. Trans. Am. Math. Soc. 284, 651-664 (1984)

17. Gundersen, G: Estimates for the logarithmic derivative of a meromorphic function, plus similar estimates. J. Lond. Math. Soc. 37(2), 88-104 (1988)

18. Barry, PD: On a theorem of Besicovitch. Q. J. Math. 14(1), 293-302 (1963)

19. Barry, PD: Some theorems related to the $\cos \pi \rho$ theorem. Proc. Lond. Math. Soc. 21(3), 334-360 (1970)

20. Gundersen, G: Finite order solutions of second order linear differential equations. Trans. Am. Math. Soc. 305(1), 415-429 (1988)

\section{Submit your manuscript to a SpringerOpen ${ }^{\circ}$ journal and benefit from:}

$\checkmark$ Convenient online submission

- Rigorous peer review

Immediate publication on acceptance

- Open access: articles freely available online

- High visibility within the field

- Retaining the copyright to your article 